\title{
Recurrency of an Idiopathic Triple Transient Jejuno-Jejunal Intussusception and Acute Pancreatitis in an Adult A Case Report
}

Ovidiu-Angel Matei ${ }^{*}$, Carolin Niethammer, Wolfram Lamade, Meike-Sarah Bauer

General and Visceral Surgeon, Department of General and Visceral Surgery, Helios Hospital Pforzheim, Germany

DOI: $\underline{10.36347 / \text { sasjs.2021.v07i02.007 }}$

| Received: 11.01.2021 | Accepted: 22.01.2021 | Published: 10.02.2021

*Corresponding author: Ovidiu-Angel Matei

Abstract

Introduction: A jejunal intussusception is a rare cause of acute abdominal pain in adults, and multiple transient intussusception associated with acute pancreatitis are even more rare. Case presentation: We present the case of a $28-$ year-old gentleman who presented in December 2017 to our emergency department complaining of sever acute epigastric abdominal pain, radiating to the left upper quadrant. He mentionated drinking alcohol a day before. The blood tests illustrated raised amylase and lipase, Alcohol (Promille) was negativ. CT of abdomen also releaved double jejuno-jejunal intussusception distal from Treitz, no other pathological signs. Diagnostic laparoscopy revealed an intussusception of the proximal jejunum amount ligament of Treitz. A reduction of the intussusception was not successful. A diagnosis of jejuno-jejunal intussusception amount of Ligament of Treitz, also four more additional concomitant intussusception distal to it was confirmed during median laparotomy. A manually examination of the entire small bowel was then performed an allowed an easy manual reduction and therefore the aetiology of his intussusception was unknown. A bowel resection was not performed.Post-operatively the patient did well. This patient presented in January, June and September 2018 complaining of acute epigastric abdominal pain. CT showed multiple jejuno-jejunal intussusceptions amount and distal from ligament of Treitz without complications. As his symptoms had improved noticeably, he was therefore discharged home. Conclusion: An recurrent idiopathic multiple transient jejuno-jejunal intussusception and acute pancreatitis in an adult it is an extremely rare coexistence in adult. Since the symptoms of pain were persistent and the CT was demonstrating the diagnosis, the standard treatment of intussusception in adults should remain surgical.

Keywords: Triple transient jejuno-jejunal intussusception in an adult, recurrency, idiopathic, acute pancreatitis.

Copyright (C) 2021 The Author(s): This is an open-access article distributed under the terms of the Creative Commons Attribution 4.0 International License (CC BY-NC 4.0) which permits unrestricted use, distribution, and reproduction in any medium for non-commercial use provided the original author and source are credited.

\section{INTRODUCTION}

A jejunal intussusception is a rare cause of acute abdominal pain in adults, and multiple transient intussusception associated with acute pancreatitis are even more rare. Acute pancreatitis it is a frequently abdominal pain in adult, obstruction of the common bile duct by stones (38\%) and alcohol consume $(36 \%)$ it is one of the most underlying cause $[1,2]$.

A proximal jejunal intussusception at the level of ligament of Treitz prevented the normal flow of pancreatic enzymes, which may have caused the acute pancreatitis [6].

\section{CASE PRESENTATION}

We present the case of a 28-year-old gentleman who presented in December 2017 to our emergency department complaining of a 12 hour history of sever acute epigastric abdominal pain, radiating to the left upper quadrant. He had associated vomiting and diarrhea, but not fever. He did not notice any blood in his vomit or stool. He mentionated drinking alcohol a day before.

No history of previous abdominal surgery was declared by him and his family history was not contributory.

He is an active smoker, admitted to drinking alcohol every day (alcohol abuse) and to using intravenous drugs in the remote past. He smoked marijuana occasionally.

Physical examination revealed epigastric abdominal wall defense and rebound pain. The rest of physical examination was within normal limits.

The blood tests illustrated raised amylase of 322 U/L (25-125), lipase 2387 U/L (8-78), GOT-AST 
of $110 \mathrm{U} / \mathrm{L}(<35)$, GPT-ALT $72 \mathrm{U} / \mathrm{L}$ (<45), Gamma-GT of U/L ( $<60)$, Alcohol (Promille) was negativ, a white cell count of $15.6 / \mathrm{nL}(4.3-10.8)$ and D-Dimer 1.57 $\mathrm{mg} / \mathrm{L}(<0.50)$.

Ultrasound examination identified free fluid in the pouch of Douglas, and a "target" appearance on epigastric and left upper quadrant without evidence of gallstones

Contrast-enhanced computed tomography of abdomen showed a jejunal intussusception amount ligament of Treitz (Fig-1).

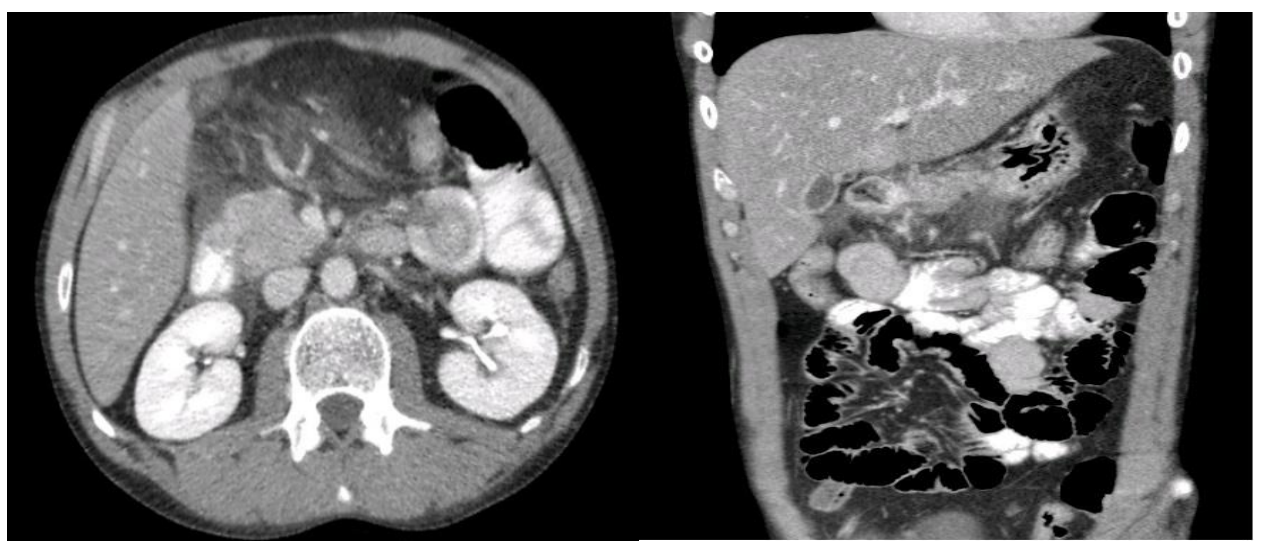

Fig-1: A jejunal intussusception amount ligament of Treitz

CT of abdomen also releaved double jejunojejunal intussusception distal from Treitz (Fig-2). There were signs of acute pancreatitis without evidence of gallstones or bowel obstruction, or perforation, no tumor, no complications of the acute pancreatitis, or entlarged lymph nodes.

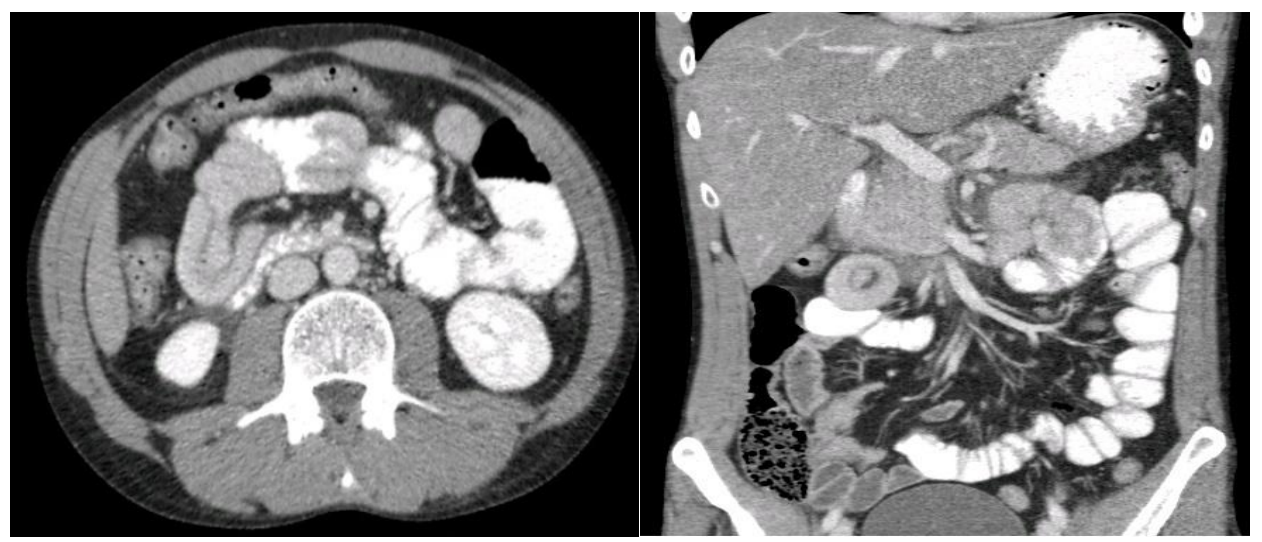

Fig-2: Double jejuno-jejunal intussusception distal from Treitz

Since the symptoms of pain were persistent and the CT was demonstrating several jejunal intussusceptions with possible presence of underlying pathology, the decision was made to take him for laparoscopic exploration.

He underwent a diagnostic laparoscopy which revealed an intussusception of the proximal jejunum amount ligament of Treitz.

A reduction of the intussusception was attempted but was not successful. We made an median epigastric laparotomy. A diagnosis of jejuno-jejunal intussusception amount of Ligament of Treitz, also four more additional concomitant intussusception distal to it was confirmed.

A manually examination of the entire small bowel was then performed an allowed an easy manual reduction. There was no evidence of ischemia or tumor or any other pathological trigger, and therefore the aetiology of his intussusception was unknown. A bowel resection was not performed.Post-operatively the patient did well and was discharged on day seven.

The patient was informed and educated about his conditions, was prescribed painkillers and antispasmodic for symptomatic relief when needed. He was instructed to return to the emergency room if an attack does not resolve.

This patient presented in January 2018 to the emergency department with recurrent history of acute abdominal pain in the left midabdomen.

The blood tests illustrated a light raised lipase 83 U/L (8-78) and Alcohol-test (Promille) was negativ. The other blood-tests were within normal limit. 
Contrast-enhanced computed tomography (Fig-3) of abdomen showed a distal jejunal intussusception without bowel obstruction, some edema of pancreas head.

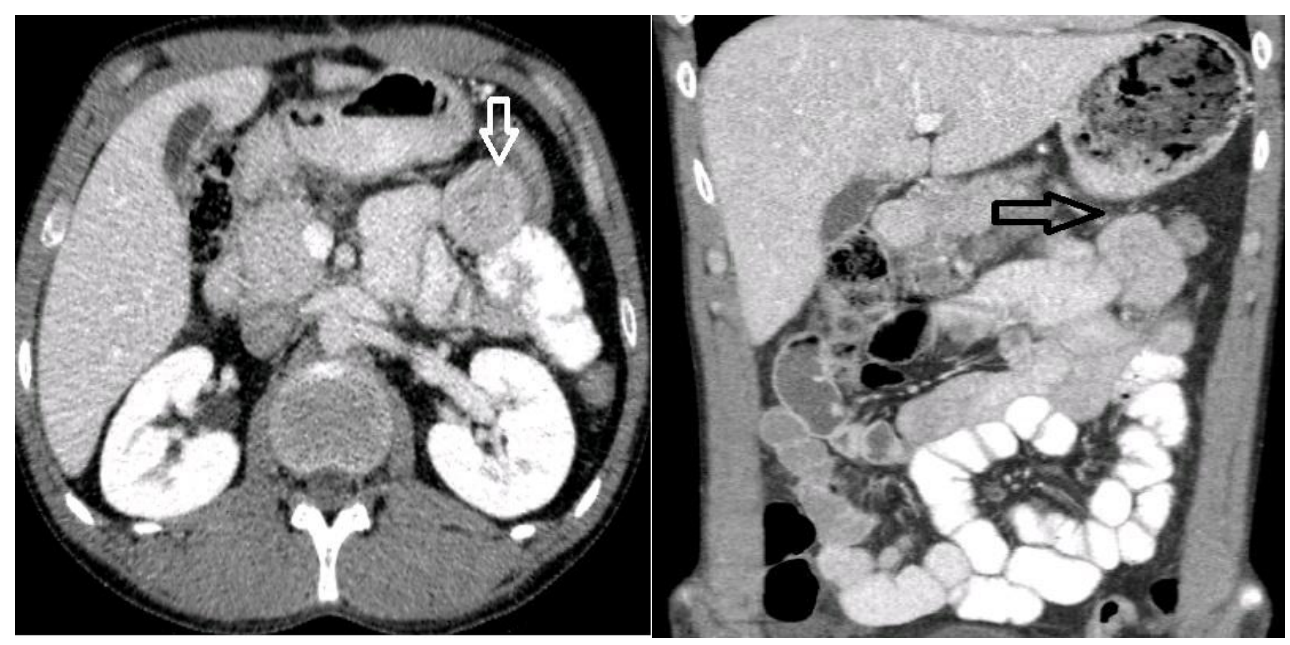

Fig-3: Distal jejunal intussusception

There were no complications and the diagnostic of jejunal intussusception with regression of an pancreatitis were made. $\mathrm{He}$ was managed symptomatically with intravenous normal saline fluids, "nothing by mouth" status, anti-spasmodic and pain killers.

As his symptoms had improved noticeably, he was therefore discharged home 2 days later with an advice to follow-up as an out-patient with his primary care physician.

The patient was admitted to the Emergency Department June 2018 with intense left-sided abdominal pain. Result of routine blood test revealed elevated lipase 5605 U/L (8-78), CRP 0.96 mg/dl (0.000.50 ) and Alcohol-test (Promille) was negativ. The other blodd tests were normal. A computed tomography image was highly suggestive of proximal jejunum intussusception amount ligament of Treitz and massive swollen pancreas with small abscess (Fig-4)

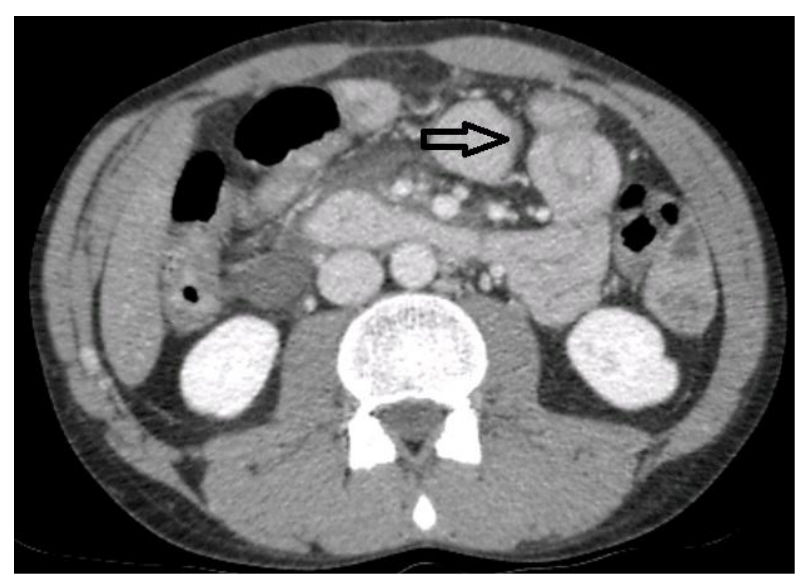

Fig-4: Proximal jejunum intussusception amount ligament of Treitz
He was managed symptomatically, his symptoms and blood tests had improved noticeably and administration of enteral nutrition was well tolerated 24-hours after admission to hospital.

He was discharged home 4 days later. In September 2018 he underwent contrast-enhanced computed tomography of the abdomen regarding recurrent left-sided abdominal pain. The examination showed triple jejuno-jejunal intussusceptions distal from ligament of Treitz without complications. The pancreatitis signs were noticeably improved (Fig-5).

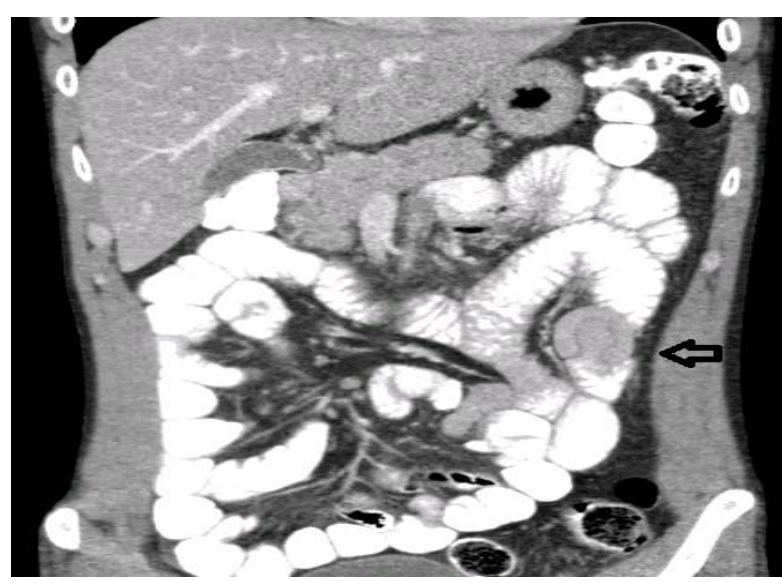

Fig-5: Jejuno-jejunal intussusceptions distal from ligament of Treitz

Laboratory evaluation showed lipase $378 \mathrm{U} / \mathrm{L}$ (8-78) and Alcohol-test (Promille) was negativ. He was managed symptomatically and discharged home 2 days later, as his symptoms had improved noticeably.

Thereafter, up to now, jejunal intussusception has not recurred. The final diagnosis was "recurrency of an idiopathic multiple transient jejunal intussusception and acute pancreatitis" 


\section{DISCUSSION}

A jejunal intussusception remain a rare cause of acute abdominal pain in adults, and multiple transient intussusception associated with acute pancreatitis are even more rare. Acute pancreatitis it is a frequently abdominal pain in adult, obstruction of the common bile duct by stones (38\%) and alcohol consume $(36 \%)$ it is one of the most underlying cause $[1,2]$.

Intussusception is on the other hand primarily a childhood disease, comparing with pancreatitis witch is almost reported in adult.

In $70 \%-90 \%$ of cases of adult intussusception, an underlying organic cause is found. This is in contrast to the pediatric population, which has an idiopathic and spontaneous form in over $95 \%$ of cases.

Recent studies show that $60 \%$ of small-bowel intussusception are caused by malignancy, the remainder of instance are caused by benign lesions $(30 \%)$ or are idiopathic $(10 \%)$.

The pathophysiology of cases without relation to a lead point is considered idiopathic, and in adults, it is typically transient. Most cases of intussusception in adults that involve only the small bowel are also idiopathic [3-5].

A proximal jejunal intussusception at the level amount ligament of Treitz prevented the normal flow of pancreatic enzymes, which may have caused the acute pancreatitis [6].

The clinical investigation and treatment is essential and also different in both of the conditions.

Clinical signs, blood tests and physical examination findings mostly help to diagnose acute pancreatitis. In patients with intussusception, however, the clinical findings may be obscure.

Contrast-enhanced CT is the most usefull modality of diagnosis for intussusception in the adult. This bowel-within-bowel appearance on CT scan is pathognomonic for intussusception. A lead point can be identified in some cases, although this is a difficult task in many patients.

With the increasing use of CT in patients with gastrointestinal symptoms, the number of adults identified with unsuspected intussusception is likely to increase [7-9].

Also CT may provide important findings of coexisting multiple intraabdominal pathologies that can easily be overlooked, as it was the issue in the presented case.

Imaging by acute pancreatitis is crucial for the detection of complications and to help guide treatment.
Most surgeons believed that laparotomy should be performed on all patients of high likehood of identifying a pathologic lesion; there are no universally accepted guidelines [10].

Because of high incidence of complications, the standard treatment of intussusception in adults is surgical, without prior nonoperative attempts at reduction [11].

Medical management of mild acute pancreatitis is relatively straightforward. The patient is kept NPO (nil per os - that is, nothing by mouth), and intravenous fluid hydration is provided. Analgesics are administered for pain relief. Antibiotics are generally not indicated.. Systematic reviews and meta-analyses have shown that administration of enteral nutrition may reduce mortality and infectious complications compared with parenteral nutrition [12].

\section{CONCLUSIONS}

An recurrent idiopathic multiple transient jejuno-jejunal intussusception and acute pancreatitis in an adult it is an extremely rare coexistence.

By the virtue of its rarity, recurrent transient intussusception in adult with acute pancreatitis are unexpected, an early diagnosis with a CT scan will identify patients requiring emergent surgery and thus prevent serious complications such as haemorrhage, intestinal gangrene and perforation. Since the symptoms of pain were persistent and the CT was demonstrating the diagnosis, the standard treatment of intussusception in adults should remain surgical.

The practitoners should be thinking about a proximal jejunal intussusception (at the level of ligament of Treitz), which may cause acute pancreatitis in adult without history of stones or alcohol abuse.

Histological evidence of recurrent multiple transient jejuno-jejunal intussusception do not exist in our archives.

\section{Author Contributions}

All authors contributed equally to the manuscript drafting. All authors read and approved the final manuscript.

\section{REFERENCE}

1. Lankisch PG, Assmus C, Lehnick D, Maisonneuve P, Lowenfels AB. Acute pancreatitis: does gender matter? Dig Dis Sci. 2001;46:2470-2474.

2. Spanier BW, Dijkgraaf MG, Bruno MJ. Epidemiology, aetiology and outcome of acute and chronic pancreatitis: An update. Best Pract Res Clin Gastroenterol. 2008; 22:45-63. 
3. Derik LD, Harvey S, Helen T. Morehouse. A 57Year-Old Woman With Bloody Diarrhea. Case Report- Medscape - Aug 16, 2017.

4. Marinis A, Yiallourou A, Samanides L, Dafnios N, Anastasopoulos G, Vassiliou I, Theodosopoulos T. Intussusception of the bowel in adults: a review. World journal of gastroenterology: WJG. 2009 Jan 28;15(4):407411.

5. Dahnert W. Radiology Review Manual. 5th ed. New York, NY: Lippincott Williams \& Wilkins; 2003:835-837.

6. Yuta Koike. A rare cause of recurrent acute pancreatitis: duodenum intussusception by a fenestrated duodenal web. American Society for Gastrointestinal Endoscopy. 2017, 86(3), 567-568

7. Kim YH, Blake MA, Harisinghani MG. Adult intestinal intussusception: CT appearances and identification of a causative lead point. RadioGraphics. 2006; 26:733-744.

8. Gayer G, Apter S, Hofmann CE, Nass S, Amitai M, Zissin R, Hertz M. Intussusception in adults: CT diagnosis. Clinical radiology. 1998 Jan 1;53(1):53-7.

9. Daniel JB, René P. Revised Atlanta classification of acute pancreatitis, 2016.

10. Takeuchi K, Tsuzuki Y, ando T, Sekihara M, Hara T, Kori T, Kuwano H. The diagnosis and treatment of adult intussusception. $\mathbf{J}$ Clin Gastroenterol. 2003; 36(1):18-21.

11. Barussaud M, Regenet N, Briennon X. Clinical spectrum and surgical approach of adult intussusception. World J Surg. 2006;30:1300-4

12. Krishnan K. Nutritional management of acute pancreatitis. Curr Opin Gastroenterol. 2017 Mar. 33(2):102-6. 